\title{
Conservación de la tortuga carey (Eretmochelys imbricata) en Rincón del Mar, Colombia
}

\section{Conservation of the hawksbill turtle (Eretmochelys imbricata) in Rincón del Mar, Colombia}

\author{
Nicolás Restrepo-Garzón ${ }^{1,2 *}$, Rafael Andrés Álvarez-Rodríguez ${ }^{1}$, Cristian Ramírez-Gallego ${ }^{3}$, \\ $\begin{array}{lll}\text { (iD) } 0000-0003-4551-4809 & \text { (iD } 0000-0002-7206-1035 & \text { (iD) } 0000-0001-9485-5736\end{array}$ \\ Tulio Ruíz-Álvarez y Karla G. Barrientos-Muñoz ${ }^{4}$ \\ $\begin{array}{ll}\text { (iD) } 0000-0003-3868-2126 & \text { (iD) } 0000-0003-3963-5727\end{array}$ \\ 1.Corporación Autónoma Regional de Sucre-CARSUCRE.Sincelejo,Colombia.rafalvarez1227@gmail.com,truiz@carsucre.gov.co \\ 2. Universidad de los Andes, Bogotá, Colombia.nicolas.r.gar@gmail.com* \\ 3. Corporación para el Desarrollo de la Costa Caribe - CORPOCARIBE, Sincelejo, Colombia.ramirezgallego.cristian@gmail.com \\ 4. Fundación Tortugas del Mar - FTM y Wider Caribbean Sea Turtle Conservation Network - WIDECAST. Envigado, Antioquia, Colombia. \\ karla.barrientos@udea.edu.co \\ * Autor de correspondencia.
}

\section{RESUMEN}

$\mathrm{S}$ e describen los procesos de conservación y ecología de anidación de la tortuga carey (Eretmochelys imbricata), producto del monitoreo sistemático entre 2018 y 2019 en el corregimiento de Rincón del Mar, San Onofre, Sucre. Se registraron seis nidadas de tortuga carey, todos fueron dejadas in situ y un nido fue saqueado por humanos. Las hembras desovaron un total de 761 huevos en cinco nidadas (promedio \pm DE: $152,2 \pm 15,5$ huevos por nidada). El éxito de eclosión fue en promedio $78,2 \pm 12,4 \%$ y el éxito de emergencia fue en promedio $74,7 \pm 14,1 \%(n=5)$. El periodo de incubación fue en promedio de $54 \pm 7,8$ días $(n=4)$. Fueron liberadas 591 crías al mar, de las cuales 22 (3,72\%), fueron rescatadas y liberadas con la comunidad. Además, se realizaron mediciones físicas de los nidos (ancho, profundidad, distancia a la playa), medidas morfométricas de los neonatos (ARC, LRC y peso) y se identificaron amenazas en el sector. Nuestros resultados contribuyen al conocimiento del estado actual de la tortuga carey, así como lo indispensable de la participación comunitaria para su conservación en Rincón del Mar.

PALABRAS CLAVE: tortuga carey, conservación, ecología de anidación, éxito de eclosión, Caribe colombiano.

\section{ABSTRACT}

$\mathrm{T}$ The conservation and nesting ecology of the hawksbill turtle (Eretmochelys imbricata) are described, as a result of a systematic monitoring between 2018 and 2019 in the municipality of Rincón del Mar, San Onofre, Sucre. Six hawksbill turtle nests were recorded in situ, one nest was poached by humans. The nesting female spawned a total of 761 eggs in five clutches (mean $152.2 \pm 15.5$ eggs per clutch). Mean hatching success was $78.2 \pm 12.4 \%$ and the mean emergence success was $74.7 \pm 14.1 \%(n=5)$. The mean incubation period was $54 \pm 7.8$ days $(n=4) .591$ hatchlings were released into the sea, of which $22(3.72 \%)$ were rescued and released with the community. In addition, physical measurements of the nests (width, depth, distance to the beach) and morphometric measurements of the hatchlings (SCW, SLC and weight) were carried out. Finally, 14 natural and anthropogenic threats were identified for the species in Rincón del Mar and different degrees of threat, according to the beach sector, being high, medium and low for Chichimán, Ana Gómez and Balsillas with Punta Gorda respectively. Our results contribute to the knowledge of the current status of the hawksbill turtle, as well as the importance of the community participation, systematic and standardized monitoring, and the urgency of generating plans and management units for their conservation in Rincón del Mar.

KEYWORDS: hawksbill turtle, conservation, nesting ecology, hatching success, Colombian Caribbean.

DOI: https://doi.org/10.25268/bimc.invemar.2021.50.2.1066 


\section{INTRODUCCIÓN}

La tortuga carey (Eretmochelys imbricata) se encuentra categorizada En Peligro Crítico de extinción por La Unión Internacional para la Conservación de la Naturaleza (UICN) y en el libro Rojo de reptiles de Colombia (Meylan y Donelly 1999; Mortimer y Donelly, 2008; Barrientos-Muñoz et al., 2015). Adicionalmente, se encuentra en el Apéndice I de la Convención sobre el Comercio Internacional de Especies Amenazadas de Fauna y Flora Silvestres, CITES (Rhodin et al., 2018), en los Apéndices I y II de la Convención sobre la Conservación de las Especies Migratorias de Animales Silvestres, CMS, y en el Anexo II del Protocolo Relativo a las Áreas y Flora Fauna Silvestres Especialmente Protegidas, SPAW (BarrientosMuñoz et al., 2020).

Sin embargo, a pesar de estar protegida por leyes $\mathrm{y}$ tratados nacionales e internacionales, es la tortuga marina con mayor presión antrópica (Meylan y Donelly 1999; Mortimer y Donelly, 2008; Barrientos-Muñoz et al., 2015). Además del consumo de su carne y huevos, el tráfico ilegal de su caparazón para la elaboración de artesanías, utensilios de cocina y espuelas para peleas de gallos, es la principal razón de su estado crítico a nivel mundial y local (Meylan, 1999; Reuter y Allan, 2006; Barrientos-Muñoz et al., 2015, 2020; Ramírez-Gallego y Barrientos-Muñoz, 2020, 2021 ).

En Colombia, la tortuga carey es la especie con mayor distribución en playas de anidación en el mar Caribe, teniendo presencia en todos los departamentos de la cuenca, pero con la menor abundancia de nidos por temporada y sin estudios poblacionales exclusivos para la especie (CeballosFonseca, 2004; Barrientos-Muñoz et al., 2015). Los aportes al conocimiento de la tortuga carey están relacionados con avistamientos en sitios de anidación y/o en el agua de manera esporádica, mientras se monitorean otras especies, no necesariamente tortugas marinas (McCormick, 1997, 1998; Rincón et al., 2001; Arcos et al., 2002; CeballosFonseca, 2004; Rincón-Díaz y Rodríguez-Zárate, 2004; Barrientos-Muñoz et al., 2015).

Ahora bien, aunque no existen estudios poblacionales de la especie en Rincón del Mar, en el Caribe colombiano la temporada de anidación de la tortuga carey se extiende desde abril hasta noviembre (Kaufmann, 1967) con dos picos de anidación en mayo y septiembre (BarrientosMuñoz et al., 2015). En la actualidad el departamento con mayor anidación de la tortuga carey en Colombia es el Archipiélago de San Andrés, Providencia y Santa Catalina

\section{INTRODUCTION}

The hawksbill turtle (Eretmochelys imbricata) is classified as critically endangered by the International Union for the Conservation of Nature (IUCN) and the Libro rojo de reptiles de Colombia [Red book of Colombian reptiles] (Meylan and Donelly 1999; Mortimer and Donelly, 2008; Barrientos-Muñoz et al., 2015). In addition, the species features in Appendix I of the Convention on International Trade in Endangered Species of Wild Fauna and Flora, CITES (Rhodin et al., 2018), Appendices I and II of the Convention on the Conservation of Migratory Species (CMS) and in Appendix II of the Protocol for Specially Protected Areas and Wildlife, SPAW (Barrientos-Muñoz et al., 2020).

However, despite the fact it is protected by legislation and by national and international treaties, it is the marine turtle species that faces the highest levels of anthropic pressure (Meylan and Donelly 1999; Mortimer and Donelly, 2008; Barrientos-Muñoz et al., 2015). In addition to the consumption of its meat and eggs, the principal reason for its critical status both internationally and locally is illegal trafficking of its shell for the production of artisan products, kitchen utensils and cock fighting spurs (Meylan, 1999; Reuter and Allan, 2006; Barrientos-Muñoz et al., 2015, 2020; Ramírez-Gallego and Barrientos-Muñoz, 2020, 2021).

The hawksbill turtle is the most widely distributed marine turtle species found on Colombia's nesting beaches -although few specific population studies for the species have been conducted - and is found in all the departments comprising the Caribbean basin, though it is less abundant in seasonal nesting sites (Ceballos-Fonseca, 2004; BarrientosMuñoz et al., 2015). Contributions to our understanding of the hawksbill turtle are principally associated with sightings in nesting areas and/or, sporadically, in the water during the monitoring of other species, not necessarily marine turtles (McCormick, 1997, 1998; Rincón et al., 2001; Arcos et al., 2002; Ceballos-Fonseca, 2004; Rincón-Díaz and RodríguezZárate, 2004; Barrientos-Muñoz et al., 2015).

Although no population studies of the species have been carried out in Rincón del Mar, it is known that the nesting season of the hawksbill turtle in the Colombian Caribbean extends from April to November (Kaufmann, 1967), with two peak periods in May and September (Barrientos-Muñoz et al., 2015). Currently, the Colombian department with the greatest number of hawksbill turtle nests is the Archipelago of San Andrés, Providencia and Santa Catalina (Eckert and 
(Eckert y Eckert, 2019; Ramírez-Gallego y BarrientosMuñoz, 2020b; Barrientos-Muñoz et al., 2020).

Por su lado, en la subregión de Morrosquillo, departamento de Sucre, históricamente se han registrado de manera esporádica nidos de la tortuga carey en El Francés, isla Palma, Punta Seca, Balsillas y Altos de Julio (Rueda, 1987; Ceballos-Fonseca, 2004; Caraballo et al., 2008; Duque et al., 2011; Barrientos-Muñoz et al., 2015). Además de las zonas de anidación, la presencia de extensas praderas de pastos marinos y arrecifes coralinos ha permitido identificar múltiples etapas de vida de las tortugas marinas en zonas de alimentación, descanso y/o reproducción (Rincón-Díaz y Rodríguez-Zárate, 2004; Moncada et al., 2019). La diversidad de hábitats marinos y playas son cruciales para la conservación de tortugas marinas en esta amplia región (Rincón-Díaz y Rodríguez-Zárate, 2004; Moncada et al., 2019). Sin embargo, la pesca dirigida para el uso de su caparazón, consumo y venta de carne y/o huevos, pérdida de hábitat, erosión costera y la contaminación por plásticos son algunas de las principales amenazas que enfrentan las tortugas carey (Caraballo et al., 2008; Barrientos-Muñoz et al., 2015,2020 ).

La Unidad Ambiental Costera Estuarina del Río Sinú y Golfo de Morrosquillo, como unidad de planificación costera, incluye áreas protegidas a nivel nacional y regional que integran Subsistema de Áreas Marinas Protegidas (SAMP): PNN Corales del Rosario y San Bernardo, PNN Corales de Profundidad, DRMI Bahía Cispata, La Balsa, Tinajones y sectores vecinos al delta río Sinú, PNR Boca de Guacamayas, DRMI Ciénaga de la Caimanera y, en proceso de declaratoria, el DRMI Sabanetica, Chichimán, Rincón del Mar y Berrugas (Carsucre) (Figura 1). Dando cumplimiento a las metas Aichi de aumentar 10\% las áreas protegidas para el año en curso y permitiendo fortalecer el Sistema Nacional de Áreas Protegidas (SINAP) y la Política Nacional Ambiental para el Desarrollo Sostenible de los Espacios Oceánicos y Zonas Costeras e Insulares de Colombia (PNAOCI), lo cual se convierte en un mecanismo para la protección de la biodiversidad marina y costera, incluidas las tortugas marinas (INVEMAR-CVS, 2012; SIRAP, 2013; Cardique et al., 2016).
Eckert, 2019; Ramírez-Gallego and Barrientos-Muñoz, 2020b; Barrientos-Muñoz et al., 2020).

For its part, in the sub-region of Morrosquillo, department of Sucre, historically the hawksbill turtle has been recorded as nesting sporadically in El Francés, Isla Palma, Punta Seca, Balsillas and Altos de Julio (Rueda, 1987; Ceballos-Fonseca, 2004; Caraballo et al., 2008; Duque et al., 2011; Barrientos-Muñoz et al., 2015). In addition to the nesting zones, the presence of extensive meadows of marine grass and coral reefs has enabled the identification of multiple life stages of marine turtles in their feeding, resting and/or reproduction zones (Rincón-Díaz and RodríguezZárate, 2004; Moncada et al., 2019). A diversity of marine and beach habitats is vital to the conservation of marine turtles in this extensive region (Rincón-Díaz and RodríguezZárate, 2004; Moncada et al., 2019). However, hunting to obtain shells or for the consumption or sale of meat and/ or eggs, habitat loss, coastal erosion and contamination by plastics are among the principal threats facing the species (Caraballo et al., 2008; Barrientos-Muñoz et al., 2015, 2020).

The Unidad Ambiental Costera Estuarina del Río Sinú y Golfo de Morrosquillo is a coastal planning unit that includes national and regional protected areas. Together, these protected areas make up the Subsistema de Áreas Marinas Protegidas (Sub-system of Protected Marine Areas - SAMP): PNN Corales del Rosario y San Bernardo, PNN Corales de Profundidad, DRMI Bahía Cispata, La Balsa, Tinajones and neighboring sectors to the delta of the Sinú river, PNR Boca de Guacamayas, DRMI Ciénaga de la Caimanera and the DRMI Sabanetica, Chichimán, Rincón del Mar and Berrugas (Carsucre) (Figure 1). In fulfilment of the Aichi Target to increase protected areas by $10 \%$ by the current year this will strengthen the National System of Protected Areas (SINAP) and the National Environmental Policy towards Sustainable Development of Oceanic Spaces, Coastal and Island Regions of Colombia (PNAOCI), which will create a mechanism for the protection of marine and coastal biodiversity, including marine turtles (INVEMARCVS, 2012; SIRAP, 2013; Cardique et al., 2016). 


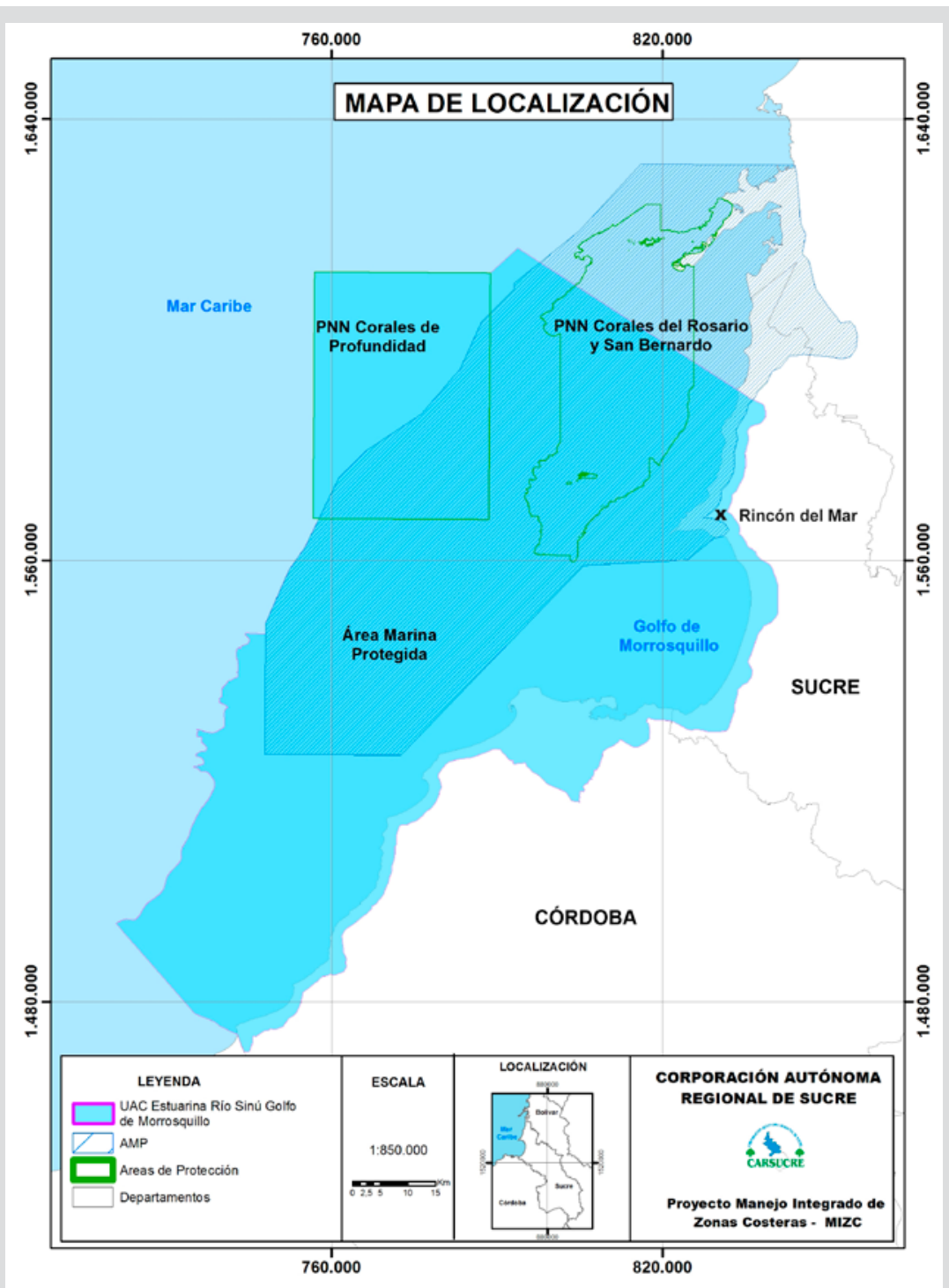

Figura 1. Subsistema de Áreas Marinas Protegidas (SAMP) presentes en el golfo de Morrosquillo.
Figure 1. Subsystem of Marine Protected Areas (SAMP) present in the Gulf of Morrosquillo.
Desde hace cinco años la Corporación Autónoma Regional de Sucre, Carsucre, ha realizado esfuerzos en control y vigilancia para reducir el uso de redes tortugueras en su jurisdicción. En isla Boquerón han sido identificados puntos de caladeros para la captura dirigida de tortugas marinas (Chelonia mydas y Eretmochelys imbricata) para su consumo y comercialización (Carsucre , 2017). Por lo cual, continuar con esfuerzos de control, vigilancia y aportes para el conocimiento de las tortugas marinas en Sucre es urgente.

Después de una década, en 2018, se registran de nuevo nidos de tortuga carey para Rincón del Mar. Por esta razón, se implementa en el marco del proyecto "Manejo integrado de la UAC estuarina río Sinú-Golfo de Morrosquillo" de Carsucre y la Fundación Tortugas del
For the last five years, the Autonomous Regional Corporation of Sucre, Carsucre, the body responsible for environmental protection in the study area, has sought to implement control and monitoring measures in order to reduce the use of turtle nets in its area of jurisdiction. Fishing grounds dedicated to the capture of marine turtles (Chelonia mydas, the Green Sea Turtle, and Eretmochelys imbricata) for human consumption and commercialization have been found in the island of Boquerón (Carsucre, 2017). It is, therefore, of urgent importance to continue with control and monitoring efforts and to contribute to increasing understanding of marine turtles in the department of Sucre.

After a decade of absence, nine hawksbill nests were recorded in Rincón del Mar in 2018. This led to the 
Mar el proceso de fortalecimiento comunitario, educación ambiental y monitoreo sistemático de la anidación de la tortuga carey en Rincón del Mar. Durante las temporadas de anidación de 2018 y 2019 se realizó el monitoreo sistemático para aportar al conocimiento de la ecología de anidación de la tortuga carey en Rincón del Mar.

\section{ÁREA DE ESTUDIO}

Rincón del Mar ( $9^{\circ} 46,5^{\prime}, 23^{\prime}$ N $, 75^{\circ} 38,31^{\prime}, 30^{\prime}$ O) se encuentra localizado en el municipio de San Onofre, Sucre (Figura 2). Presenta un clima seco, con temperaturas entre $24^{\circ} \mathrm{C}$ y $38^{\circ} \mathrm{C}$, con precipitaciones que oscilan entre 800 y $1000 \mathrm{~mm}$ anuales y un régimen de lluvias bimodal. Con dos períodos secos de diciembre a abril y junio a julio, los meses restantes son lluviosos (Cusado-Zapa y González -Pérez, 2010). implementation by Carsucre and the Fundación Tortugas del Mar of the project "Integrated management of the UAC River Sinú-Gulf of Morrosquillo estuarine", this project involves community strengthening, environmental education and the systematic monitoring of hawksbill turtle nesting sites in Rincón del Mar. Systematic monitoring was conducted during the 2018 and 2019 nesting seasons in order to contribute to understanding of the ecology of the species' nesting in the area.

\section{STUDY AREA}

Rincón del Mar $\left(9^{\circ} 46,5^{\prime}, 23^{\prime}\right.$ N, $75^{\circ}$ 38,31’,30”W) is located in the municipality of San Onofre, department of Sucre (Figure 2). It has a dry climate, with temperatures that range between $24^{\circ} \mathrm{C}$ and $38^{\circ} \mathrm{C}$ and annual rainfall that oscillates between 800 and $1000 \mathrm{~mm}$. It has a bimodal rainfall regime, with two dry periods between December and April, and June and July; the other months are dry (Cusado-Zapa and González-Pérez, 2010).

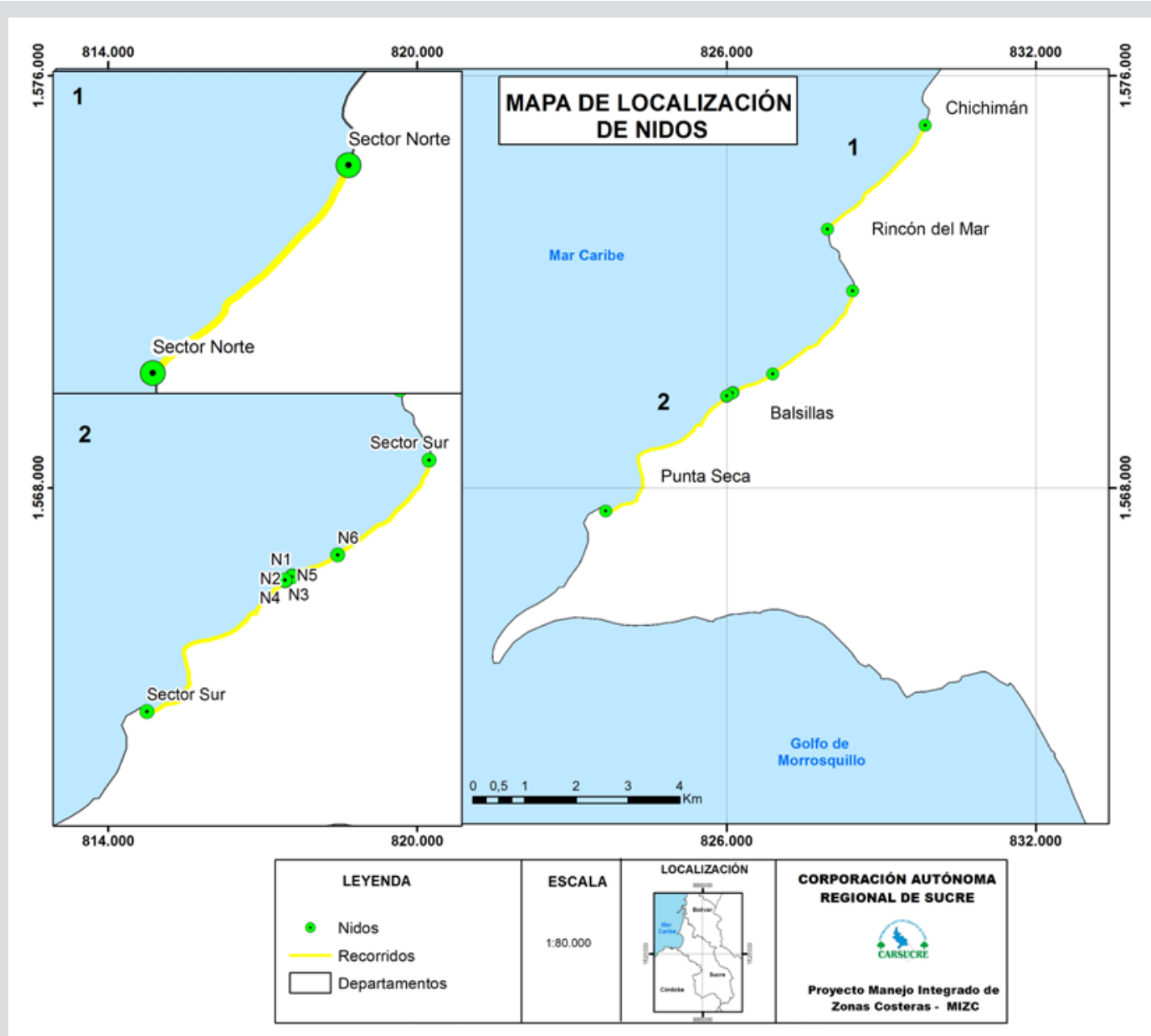

Figura 2. Área de estudio y localización de los nidos de la tortuga carey.

Figure 2. Study area and location of hawksbill nests. 
$\mathrm{Su}$ litoral costero se caracteriza por tener playas intermedias, con alta energía de oleaje y poca pendiente. La arena tiene tonalidades cremosas-pardas y están compuestas por materiales carbonatados de tamaño grueso, $27,7 \%(\mathrm{DE}=0,5 \mathrm{~mm})$, y mediano, $56 \%(\mathrm{DE}=0,25 \mathrm{~mm})$ (Caraballo et al., 2008).

En esta área se presentan asentamientos humanos destinados principalmente a la actividad turística y en algunos lugares persisten ecosistemas de manglar dominados por mangle rojo (Rhizophora mangle) y mangle blanco (Laguncularia racemosa), además de otras especies como uvita de playa (Coccoloba uvifera), clemón (Thespesia populnea), lirio de playa (Hymenocallis caribaea) y almendro (Terminalia catappa), con una distribución más limitada (Ulloa et al., 2016). En el área marina circundante se encuentran ecosistemas conformados por praderas de fanerógamas y arrecifes de coral, que han sido identificados como zonas de alimentación y reproducción de las tortugas marinas que transitan por el área (Rincón-Díaz y Rodríguez-Zárate, 2004).

\section{MATERIALES Y MÉTODOS}

\section{Monitoreo}

Se realizaron patrullajes diurnos y nocturnos entre julio-septiembre de 2018 y 2019 (meses en los cuales hay una mayor probabilidad de anidación de acuerdo con los expertos locales). Los patrullajes se dividieron en dos sectores, el sector norte comprendido entre las playas de Punta Rincón y Chichimán ( $9^{\circ} 46^{\prime} 26,43^{\prime \prime} \mathrm{N}, 75^{\circ} 38^{\prime} 43,54^{\prime \prime} \mathrm{O}$ - $\left.9^{\circ} 47^{\prime} 32,43^{\prime \prime} \mathrm{N}, 75^{\circ} 37^{\prime} 41,76^{\prime \prime} \mathrm{O}\right)$ con una extensión de $2,8 \mathrm{~km}$. El segundo sector corresponde a la parte sur entre Balsillas y la boca de Ana Gómez $\left(9^{\circ} 45^{\prime} 47,53^{\prime \prime} \mathrm{N}\right.$, $\left.75^{\circ} 38^{\prime} 27,36^{\prime \prime} \mathrm{O}-9^{\circ} 43^{\prime} 27,66^{\prime \prime} \mathrm{N}, 75^{\circ} 41^{\prime} 3,95^{\prime \prime} \mathrm{O}\right)$ con una extensión de $6,9 \mathrm{~km}$. El sector norte se monitoreó durante un corto tiempo debido a la escasa actividad de las tortugas y problemas de orden público (Figura 2). Los patrullajes nocturnos se realizaron entre 20:00 y 23:00 h en búsqueda de rastros y/o hembras anidantes. Los patrullajes diurnos se realizaron entre 5:00 y 8:00 h para el registro de huellas de tortugas en caso de no haber avistado en la noche anterior y verificar los eventos de anidación exitosos.

Registro y protección de nidos

Todos los nidos registrados fueron dejados in situ y vigilados diariamente en horas de la noche y en las mañanas. Al momento de identificar un nido, éste se señalizaba, se registraba su ubicación geográfica con un
Its coastal littoral zone is characterized by shallow intermediate beaches and high energy waves. The sand is white-gray in tone and is composed of $27.7 \%$ large-grained $(\mathrm{DE}=0,5 \mathrm{~mm})$ and $56 \%$ medium $(\mathrm{DE}=0.25 \mathrm{~mm})$ carbonate materials (Caraballo et al., 2008).

Human settlement in the area is dedicated principally to tourism and in some places mangrove ecosystems persist, dominated by red and white mangroves (Rhizophora mangle and Laguncularia racemose) and by other less widely distributed species such as the sea grape (Coccoloba uvifera), portia tree (Thespesia populnea), Caribbean spider lily (Hymenocallis caribaea) and tropical almond (Terminalia catappa) (Ulloa et al., 2016). In the surrounding maritime area, ecosystems are found consisting of meadows of phanerogams and coral reefs, which have been identified as feeding and reproduction zones for the marine turtles that pass through the area (Rincón-Díaz and Rodríguez-Zárate, 2004).

\section{MATERIALS AND METHODS}

\section{Monitoring}

Daytime and nocturnal patrols were carried out between July and September 2018 and 2019 (the months in which the likelihood of nesting was greatest, according to local experts). The patrols took place in two sectors. The northern sector, $2.8 \mathrm{~km}$ long, comprised of the beaches of Punta Rincón and Chichimán $\left(9^{\circ} 46^{\prime} 26.43^{\prime \prime} \mathrm{N}, 75^{\circ} 38^{\prime}\right.$ 43.54 ” W - $9^{\circ} 47^{\prime} 32.43^{\prime \prime} \mathrm{N}, 75^{\circ} 37^{\prime} 41.76^{\prime}$ ' W), while the second, $6.9 \mathrm{~km}$ long, corresponded to the area between Balsillas and Boca de Ana Gómez ( $9^{\circ} 45^{\prime} 47.53^{\prime \prime} \mathrm{N}, 75^{\circ} 38^{\prime}$

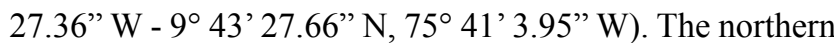
sector was only monitored for a short period due to low levels of activity of the turtles and public order problems (Figure 2). The nocturnal patrols were carried out between 20:00 and 23:00 $\mathrm{h}$ and looked for tracks and/or laying females. The daytime patrols were carried out between 05:00 and 08:00 $\mathrm{h}$ and were intended to record turtle tracks that had not been found the night before, with intention of verifying successful nesting events.

Recording and protecting nests

All the nests recorded were left in situ and monitored daily at night and in the mornings. When a nest was identified, signs were posted, its location was registered using GPS (Gpsmap 64s), along with the time and date the eggs were laid and the distance of the nest from the high 
GPS (Gpsmap 64), la hora y fecha de puesta, y la distancia al punto más alto de marea. Posteriormente, los nidos se protegieron con encierros de malla plástica con dimensiones de 2 x $2 \mathrm{~m}$ y $60 \mathrm{~cm}$ de alto. A las nidadas que en el transcurso del periodo de incubación presentaron evidente exposición al oleaje, se les construyeron barreras con sacos rellenos de arena de la misma playa, la cual era nuevamente depositada al culminar el proceso.

Luego de 40 días de incubación, se revisaron varias veces al día, para inspeccionar evidencia de emergencia de las crías. Cuando se observaron huellas de crías salidas de un nido, se esperaron varias horas y hasta un máximo de 24 horas para realizar la excavación.

\section{Productividad de las nidadas}

Posterior a que las crías emergieran de los nidos hacia el mar, se esperaron $24 \mathrm{~h}$ para realizar la excavación y determinar el éxito de eclosión y de emergencia. Durante las excavaciones se contaron los cascarones vacíos (> $50 \%$ completa) (C), huevos sin desarrollo aparente (HSDA), embriones a término no eclosionados (ETNE), huevos depredados (D), crías vivas encontradas atrapadas y/o por debajo del cuello del nido (V), crías muertas que lograron salir del cascarón (M) y huevos no eclosionados (HNE) (Miller, 1999), los cuales se clasificaron dentro de cuatro categorías de estadios embrionarios (Chacón et al., 2007).

Se estimó el éxito de la eclosión utilizando la fórmula: Éxito de Eclosión $(\%)=\mathrm{C} /(\mathrm{C}+\mathrm{HSDA}+\mathrm{HNE}+$ ETNE + D) * 100. A su vez, se calculó el éxito de emergencia usando la fórmula, Éxito Emergencia (\%) $=\mathrm{C}-(\mathrm{V}+\mathrm{M}) /(\mathrm{C}$ + HSDA + HNE + ETNE + D) * 100 (Miller, 1999).

Las crías vivas atrapadas dentro del nido fueron rescatadas y liberadas en horas frescas de la madrugada o al anochecer en compañía de la comunidad de Rincón del Mar. Luego de la exhumación se midió la profundidad, ancho y distancia a la línea de marea de cada nido. Además de anotaciones en campo sobre la flora y fauna asociada a la nidada.

\section{Biometría de crías}

Se tomaron diez crías por nidada, a las cuales se les registró el peso con una balanza digital (Digital Pocket MH-500; precisión: 0,1 g), y con un calibrador pie de rey plástico de $15 \mathrm{~cm}$ (CLP06U; precisión: 0,05 mm), fueron tomados los datos biométricos: largo recto del caparazón (LRC), ancho recto del caparazón (ARC) (Chacón et al., 2007) y posteriormente fueron liberadas. tide line. Subsequently the nests were protected using $2 \mathrm{~m}$ square plastic cages, $60 \mathrm{~cm}$ in height. Nests that were clearly vulnerable to wave damage during the incubation period were protected by barriers made out of sandbags filled with sand from the beach, which was spread out again after the nest was vacated.

After day 40 of incubation, nests were inspected several times a day, to look for evidence of hatching. When signs were identified that hatchlings had left the nest, a few hours - up to a maximum of 24 - were left before beginning excavation.

\section{Clutch productivity}

Twenty-four hours after the hatchlings emerged and made their way to the sea, the nest site was excavated in order to determine hatching and emergence success. During the excavation process empty shells were counted ( $>50 \%$ complete) (S), as were unhatched eggs without any obvious embryonic development (UD), unhatched full-term embryos (UHT), depredated eggs (P), living hatchlings that were found trapped in the nest and/or below the neck (L), dead hatchlings that had succeeded in exiting the shell (D) and unhatched eggs (UH) (Miller, 1999). These latter were classified according to four categories of embryo development (Chacón et al., 2007).

Hatching success was estimated using the following formula: Hatching Success $(\%)=\mathrm{S} /(\mathrm{S}+\mathrm{UD}+\mathrm{UH}+\mathrm{UHT}$ $+\mathrm{P}) * 100$. Similarly, the following formula was used to calculate Emergence Success $(\%)=\mathrm{C}-(\mathrm{L}+\mathrm{D}) /(\mathrm{S}+\mathrm{UD}+$ $\mathrm{UH}+\mathrm{UHT}+\mathrm{P}) * 100$ (Miller, 1999).

Living hatchlings found within the nest were rescued and released in the cool of the early hours of the morning or at sunset, with the participation of the Rincón del Mar community. After the excavation process was completed, the depth and width of nests were measured along with their distance from the tide line. In addition, field notes were taken of the flora and fauna associated with the nest.

\section{Hatchling biometry}

Ten hatchlings were selected from each clutch. These were weighed using digital scales (Digital Pocket MH-500; precision: $0.1 \mathrm{~g}$ ) and straight carapace length (SCL) and straight carapace width (SCW) measured using a $15 \mathrm{~cm}$ caliper gauge (Rey Plastic, CLP06U; precision: $0.05 \mathrm{~mm}$ ) (Chacón et al., 2007). The hatchlings were subsequently released. 


\section{Educación ambiental}

Se conformaron dos grupos de trabajo con expertos locales para el monitoreo participativo en los sectores sur y norte. Además, se realizaron talleres de sensibilización, fortalecimiento, liberación de tortugas y limpiezas de playa incluyendo a la comunidad de Rincón del Mar.

\section{Amenazas}

Las amenazas naturales y antrópicas se identificaron mediante los recorridos en campo e información suministrada por Carsucre en sus labores de control y vigilancia de la zona. Los datos obtenidos como tensores fueron registrados en una tabla y evaluados en una escala de cero a tres grados (Ninguno: 0, Bajo: 1, Medio: 2, Alto: 3), de acuerdo con la metodología realizada por Rincón- Díaz y Rodríguez-Zárate (2004).

\section{RESULTADOS}

Monitoreo

Durante los dos años de monitoreo se emplearon 246 días (julio-octubre) con un esfuerzo de $6 \mathrm{~h} /$ día para un total de $1476 \mathrm{~h}$ para los patrullajes diurnos y nocturnos durante las temporadas de anidación 2018 y 2019.

Registro y protección de nidos

Se registraron en total seis nidos de tortuga carey, de los cuales cinco fueron registrados en 2018 y uno en 2019 , entre los meses de julio-septiembre. Un nido (20\%) fue saqueado en 2018 para su consumo. Mientras que en la temporada 2019 no se presentó saqueo de nidadas. Las playas frecuentadas por las hembras anidantes fueron las del sector sur (entre Balsillas y la Boca de Ana Gómez), con $100 \%$ de las nidadas desovadas (Figura 2).

Productividad de las nidadas

Un total de 761 huevos fueron desovados con un promedio de 152,2 $\pm 15,5$ huevos por nidada $(\mathrm{n}=5$; Tabla 1). Se registraron 591 crías liberadas al mar, de las cuales 569 crías emergieron por sí solas y 22 crías (3,72\%) fueron rescatadas de entre raíces, huevos y cascarones.

El promedio del éxito de eclosión fue 78,2 $\pm 12,4 \%$ (rango 62,8 - 91,0; $\mathrm{n}=5$ ) (Tabla 1) y el promedio del éxito de emergencia fue $74,7 \pm 14,1 \%$ (rango 59,3 - 89,7; $\mathrm{n}=5$ ) (Tabla 1). El período de incubación para la emergencia promedio fue de $54 \pm 7,8$ días (rango 47 - 63; n =4) (Tabla 1).

\section{Environmental education}

Working groups of local experts were set up, with responsibility for participatory monitoring of the northern and southern sectors. Workshops were also organized with the Rincón del Mar community, focused on awarenessraising, community strengthening, turtle release, and beach cleaning.

\section{Threats}

Natural and anthropic threats were identified on the basis of field visits and information provided by Carsucre, fruit of its control and monitoring activities in the zone. The tensor data obtained was recorded in a table and evaluated using a scale from zero to three (None: 0, Low: 1, Medium: 2, High: 3), using the method proposed by Rincón-Díaz and Rodríguez-Zárate (2004).

\section{RESULTS}

\section{Monitoring}

The two-year research process involved 246 person days of six h each (July to October), for a total during the 2018 and 2019 nesting seasons of $1476 \mathrm{~h}$ dedicated to daytime and nocturnal patrols.

Recording and protecting nests

A total of six hawksbill turtle nests were recorded, five in 2018 and one in 2019, all between July and September. One nest (20\%) was raided in 2018 and its eggs taken for food, while no clutches were lost in 2019. The beaches visited by laying females were all in the southern sector (between Balsillas and Boca de Ana Gómez); eggs were laid in all nests (Figure 2).

\section{Clutch productivity}

A total of 761 eggs were laid, with an average of $152.2 \pm 15.5$ eggs per nest $(n=5$; Table 1$)$. A total of 591 hatchlings reached the sea, of which 569 made it alone. 22 hatchlings (3.72\%) were rescued from entanglement among roots, eggs and shells.

Mean hatching success was $78.2 \pm 12,4 \%$ (range $62.8-91.0 ; \mathrm{n}=5$ ) (Table 1$)$, while mean emergence success was $74.7 \pm 14.1 \%$ (range $59.3-89.7 ; n=5)$ (Table 1$)$. The mean incubation period was $54 \pm 7.8$ days (range $47-63$; =4) (Table 1). 
Tabla 1. Productividad de las nidadas de E. imbricata en Rincón del Mar, Sucre. n.r. $=$ no registrado.
Table 1. Productivity of the nests of E. imbricata in Rincón del Mar, Sucre. n.r. $=$ not registered.

\begin{tabular}{|c|c|c|c|c|c|c|}
\hline $\begin{array}{l}\text { Nido / } \\
\text { Nest }\end{array}$ & $\begin{array}{c}\text { Fecha de desove / } \\
\text { Laying date }\end{array}$ & $\begin{array}{c}\text { Fecha de } \\
\text { emergencia / } \\
\text { Emergence date }\end{array}$ & $\begin{array}{c}\text { Periodo de } \\
\text { incubación (días) / } \\
\text { Incubation period (days) }\end{array}$ & $\begin{array}{l}\text { Total de huevos } \\
\text { en nidada / Total } \\
\text { eggs in clutch }\end{array}$ & $\begin{array}{c}\text { Éxito de eclosión / } \\
\text { Hatching success } \\
\qquad(\%)\end{array}$ & $\begin{array}{c}\text { Éxito de emergencia / } \\
\text { Emergence success } \\
(\%)\end{array}$ \\
\hline 1 & $25 / 07 / 2018$ & $20 / 09 / 2018$ & 48 & 160 & 90.0 & 88.1 \\
\hline 2 & $9 / 08 / 2018$ & $5 / 10 / 2018$ & 58 & 156 & 91.0 & 89.7 \\
\hline 3 & $3 / 09 / 2018$ & $4 / 11 / 2018$ & 63 & 172 & 62.8 & 59.3 \\
\hline 4 & $5 / 09 / 2018$ & $21 / 10 / 2018$ & 47 & 135 & 69.6 & 62.2 \\
\hline 5 & n.r. & $5 / 09 / 2019$ & n.r. & 138 & 77.5 & 73.9 \\
\hline
\end{tabular}

La principal mortalidad durante el periodo de incubación ocurrió en la fase de desarrollo embrionario (13 $\%$ ), mientras que los huevos sin desarrollo aparente (HSDA) representaron $8,8 \%(\mathrm{n}=5$ nidos; Tabla 2$)$.
The greatest mortality levels during incubation occurred during embryo development (13\%), while eggs without apparent development (UD) accounted for $8.8 \%$ of the total $(\mathrm{n}=5$ nests; Table 2$)$.
Tabla 2. Categorías HNE (subdividido en Estadios I, II, III y IV) y HSDA para las exhumaciones de nidadas de E. imbricata en Rincón del Mar, Sucre.
Table 2. Categories of unhatched eggs (UH) (subdivided into Stages I, II, III and IV) and eggs with no apparent development (UD) during the excavation of E. imbricata nests in Rincón del Mar, Sucre.

\begin{tabular}{|c|c|c|c|c|c|c|c|}
\hline \multirow{2}{*}{\multicolumn{2}{|c|}{ Categoría / Category }} & \multicolumn{5}{|c|}{ Nido / Nest } & \multirow{2}{*}{ Proporción / Percentage (\%) } \\
\hline & & 1 & 2 & 3 & 4 & 5 & \\
\hline \multirow{4}{*}{$\mathrm{HNE} / \mathrm{UH}$} & Estadio I / Stage I & 4 & 5 & 5 & 11 & 4 & 3.8 \\
\hline & Estadio II / Stage II & 2 & 2 & 43 & 9 & 2 & 7.6 \\
\hline & Estadio III / Stage III & 0 & 1 & 0 & 3 & 0 & 0.5 \\
\hline & Estadio IV / Stage IV & 3 & 2 & 0 & 3 & 0 & 1.1 \\
\hline & HSDA / UD & 7 & 4 & 16 & 15 & 25 & 8.8 \\
\hline
\end{tabular}

El tamaño de los nidos fue de 41,4 $\pm 5,6 \mathrm{~cm}$ de profundidad y $24,6 \pm 7,8 \mathrm{~cm}$ de ancho, con una distancia a la línea de marea de 9,7 $\pm 12,5 \mathrm{~m}(\mathrm{n}=5$; Tabla 3$)$. Las nidadas $1,2,3$ y 4 fueron desovadas entre $R$. mangle y $H$. caribaea rodeadas por un mosaico de hábitats estructurados por un complejo lagunar ubicado de forma paralela a la línea de costa, de manera que los huevos de la parte inferior de las nidadas alcanzaron a tener contacto con el nivel freático. Por su parte el nido 5 , se encontró debajo de C. uvifera y H. caribaea (Tabla $3)$. En general, se encontraron bajo sombra proporcionada por el dosel de los árboles y cubiertos de hojarasca.

La fauna silvestre observada con influencia en el área de anidación fueron principalmente aves de las especies Megaceryle torquata, Pelecanus occidentalis, Quiscalus mexicanus, Egretta tricolor, E. thula, Milvago chimachima, Sterna hirundo, Thalasseus maximus, T. sandvicensi y
In terms of size, the nests had a mean depth of 41.4 $\pm 5,6 \mathrm{~cm}$ and width of $24.6 \pm 7,8 \mathrm{~cm}$ and a distance from the tide line of $9.7 \pm 12.5 \mathrm{~m}(\mathrm{n}=5$; Table 3$)$. Clutches 1 , 2, 3 and 4 were laid in areas of R. mangle and H. caribaea. They were surrounded by a mosaic of habitats made up of a lacustrine complex lying parallel to the coastline, with the result that the lower eggs in the clutches entered into contact with the phreatic zone. For its part, nest 5 was found beneath C. uvifera and H. caribaea (Table 3). In general, nests were found in areas of shade provided by the tree canopy and beneath a layer of fallen leaves.

The fauna observed in the nesting area consisted principally of birds of the species Megaceryle torquata, Pelecanus occidentalis, Quiscalus mexicanus, Egretta tricolor, E. thula, Milvago chimachima, Sterna hirundo, Thalasseus maximus, T. sandvicensi and Fregata 
Fregata magnificens. Sobre mastofauna se presenció a la especie Procyon cancrivorus, además de rastros de animales domésticos como perros y cerdos.

Tabla 3. Características físicas y vegetación asociada a las nidadas de $E$. imbricata en Rincón del Mar, Sucre. n.r. = no registrado. magnificens. In terms of mammalian fauna, Procyon cancrivorus was found, along with domestic animals such as dogs and pigs.

\begin{tabular}{|c|c|c|c|c|}
\hline $\begin{array}{c}\text { Nido / } \\
\text { Nest }\end{array}$ & $\begin{array}{c}\text { Distancia a línea de marea / } \\
\text { Distance to tide line }(\mathbf{m})\end{array}$ & $\begin{array}{c}\text { Profundidad nido / } \\
\text { Depth of nest }(\mathbf{c m})\end{array}$ & $\begin{array}{c}\text { Ancho de fondo nido / } \\
\text { Width of nest }(\mathbf{c m})\end{array}$ & $\begin{array}{c}\text { Vegetación asociada / } \\
\text { Associated vegetation }\end{array}$ \\
\hline 1 & 3 & 37 & 21 & R. mangle y H. caribaea \\
\hline 2 & 4 & 41 & 35 & R. mangle y H. caribaea \\
\hline 3 & 6 & 40 & 14 & R. mangle y H. caribaea \\
\hline 4 & 3,5 & 51 & 25 & R. mangle y H. caribaea \\
\hline 5 & 5 & 38 & 28 & C. uvifera y H. caribaea \\
\hline
\end{tabular}

\section{Biometría de crías}

Se tomaron diez crías de una nidada con valores promedio de LRC de 4,0 $( \pm 0,4) \mathrm{cm}$, de ARC de 2,9 $( \pm 0,2)$ cm y peso total de $15,4( \pm 0,5) \mathrm{g}$ (Tabla 4$)$.

Tabla 4. Mediciones biométricas (promedio \pm DE y n) de crías de $E$. imbricata de Rincón del Mar, Sucre y otros estudios realizados en el Caribe y Pacífico. Dónde: $\mathrm{LRC}=$ largo recto del caparazón $(\mathrm{cm}), \mathrm{ARC}=$ ancho recto del caparazón $(\mathrm{cm})$ y $\mathrm{P}=$ peso $(\mathrm{g})$. n.r. $=$ no registrado.

\section{Hatchling biometry}

Ten hatchlings were selected from each clutch, with average LRC dimensions of $4.0( \pm 0.4) \mathrm{cm}$, ARC of $2.9( \pm 0.2) \mathrm{cm}$ and total weight of $15.4( \pm 0.5) \mathrm{g}$ (Table 4).

\begin{tabular}{|c|c|c|c|c|c|}
\hline LRC/ SCL (cm) & ARC / SCW (cm) & P / W (g) & \multicolumn{2}{|c|}{ Lugar / Location } & Referencia / Reference \\
\hline $4.2(25)$ & $3.1(25)$ & $15.4(25)$ & Magdalena, Colombia & Kaufmann, 1975 \\
$4.3 \pm 0.2(1138)$ & $3.1 \pm 0.1(1138)$ & $16.3 \pm 1.8(1138)$ & Limón, Costa Rica & Chacón et al., 2004 \\
\hline $4.5 \pm 0.1(29)$ & $3.2 \pm 0.1(29)$ & $17.7 \pm 0.9(29)$ & Magdalena, Colombia & Joya y Molina, 2006 \\
$3.7 \pm 0.4(492)$ & $2.5 \pm 0.4(492)$ & $17.1 \pm 2.8(492)$ & Limón, Costa Rica & Hancock, 2008 \\
$3.8 \pm 0.2(10447)$ & n.r. & $11.2 \pm 1.2(11975)$ & Bahía de Jiquilisco, El Salvador & Liles et al., 2019 \\
$3.7 \pm 0.2(17014)$ & n.r. & $11.9 \pm 1.5(16996)$ & Estero Padre Ramos, Nicaragua & Liles et al., 2019 \\
\hline $4.0 \pm 0.4(10)$ & $2.9 \pm 0.2(10)$ & $15.4 \pm 0.5(10)$ & & Sucre, Colombia & Este estudio \\
\hline
\end{tabular}

\section{Educación ambiental}

Se conformó el grupo "Guardianes de las Tortugas de Rincón del Mar" con su logo e implementos para los patrullajes diurnos y nocturnos, con el fin de fortalecer su sentido de pertenencia y establecer el monitoreo participativo comunitario. Doce personas mayores de edad hicieron parte del grupo.

Un total de ocho talleres y capacitaciones sobre la biología de las tortugas marinas, técnicas de monitoreo y manejo fueron llevados a cabo con público en general de
Tabla 4. Biometric measurements (mean $\pm \mathrm{SD}$ and $\mathrm{n}$ ) of E. imbricata hatchlings from Rincón del Mar compared to other in-situ studies on the Caribbean and Pacific coasts. Where: SCL $=$ straight carapace length $(\mathrm{cm})$, $\mathrm{SCW}=$ straight carapace width $(\mathrm{cm})$ and $\mathrm{W}=$ weight $(\mathrm{g})$. n.r. $=$ not registered . 
Rincón del Mar. Un total de 24 personas participaron de los eventos de fortalecimiento. Un taller interinstitucional e intersectorial con pares internacionales fue llevado a cabo en el corregimiento de Rincón del Mar, con la participación de 5 entidades y 28 personas.

Dos liberaciones de crías fueron realizadas con la institución educativa de Rincón del Mar y el grupo Titanes Ecológicos. Un total de 30 personas entre niños y adultos de la comunidad hicieron parte de las liberaciones. Cuatro actividades de sensibilización fueron llevadas a cabo alrededor de Rincón del Mar con volantes y afiches en puntos estratégicos: tiendas y hoteles, para desestimar el consumo de carne y huevos de la tortuga carey, además del uso de su caparazón para la realización de artesanías, utensilios de cocina y espuelas de gallo. Finalmente, dos limpiezas de playa en el sector de Punta Gorda fueron llevadas a cabo con 12 personas de Rincón del Mar y un video informativo del proyecto fue uno de los productos de divulgación del proceso.

\section{Amenazas}

En 2019 se identificó el sacrificio de una hembra anidante en el sector norte (entre las playas de Punta Rincón y Chichimán) y en 2018 el robo de una nidada en el sector sur (entre Balsillas y la Boca de Ana Gómez). Además, se identificaron 14 amenazas naturales y antropogénicas en el corregimiento de Rincón del Mar. Por su "Alto" grado de amenaza, se destacaron: la erosión costera, depredación por animales domésticos (perros y cerdos) y silvestres (zorra manglera), contaminación por residuos sólidos, obstáculos en playa, tránsito de personas y pesca destructiva (Tabla 5). Al discriminar por playas se encontró, que las playas de Chichimán presentaron un grado "Alto" de amenaza, la boca de Ana Gómez grado "Medio" y un grado "Bajo" tanto en Balsillas como en Punta Gorda (Tabla 5). techniques and management, with the participation of the general population of Rincón del Mar. A total of 24 people participated in these community strengthening processes. An interinstitutional and intersectoral workshop was organized with international peers in Rincón del Mar, with the participation of 28 individuals from five different organizations.

Two releases of hatchlings were organized, jointly with the Rincón del Mar school and the local environmental group Titanes Ecológicos. A total of 30 community members, including children and adults, took part in these release events. Four awareness-raising activities were organized in the Rincón del Mar area, involving the distribution of leaflets and posters in strategic locations such as shops and hotels, with the aim of discouraging consumption of hawksbill turtle meat and eggs and of using its shell for making artisanal pieces, kitchen utensils and cock fighting spurs. Finally, two beach cleaning events were held in the sector of Punta Gorda, with the participation of 12 residents of Rincón del Mar, and an information video about the project was produced as part of the outreach process.

\section{Threats}

In 2019 a laying female was killed in the northern sector (between the beaches of Punta Rincón and Chichimán) and in 2018 a clutch of eggs was taken in the southern sector (between Balsillas and Boca de Ana Gómez). In addition, 14 natural and anthropic threats were identified in Rincón del Mar. Coastal erosion, depredation by domestic (dogs and pigs) and wild (racoons) animals, solid waste pollution, obstacles on the beaches, human transit and destructive fishing practices were identified as representing "High" levels of threat (Table 5). A categorization of the beaches identified Chichimán beach as facing a "High" degree of risk, Boca de Ana Gómez "Medium", and Balsillas and Punta Gorda "Low" (Table 5).

Tabla 5. Valores de amenazas para E. imbricata en Rincón del Mar, Sucre.

Table 5. Threat values for E. imbricata in Rincón del Mar, Sucre.

\begin{tabular}{|c|c|c|c|c|c|c|c|}
\hline Amenazas / Threats & Balsillas & $\begin{array}{l}\text { Punta } \\
\text { Gorda }\end{array}$ & $\begin{array}{l}\text { Punta } \\
\text { Seca }\end{array}$ & $\begin{array}{l}\text { Boca Ana } \\
\text { Gómez }\end{array}$ & Chichimán & Subtotal & $\begin{array}{c}\text { Grado de amenaza / } \\
\text { Threat level }\end{array}$ \\
\hline Erosión costera / Coastal erosion & 1 & 1 & 3 & 0 & 3 & 8 & Alto / High \\
\hline $\begin{array}{l}\text { Extracción de arena / } \\
\text { Extraction of sand }\end{array}$ & 1 & 0 & 1 & 0 & 2 & 4 & Medio / Medium \\
\hline $\begin{array}{l}\text { Pérdida de m anglar / } \\
\text { Mangrove loss }\end{array}$ & 0 & 0 & 1 & 0 & 2 & 3 & Medio / Medium \\
\hline $\begin{array}{l}\text { Desarrollo costero / } \\
\text { Coastal development }\end{array}$ & 1 & 0 & 3 & 0 & 1 & 5 & Medio / Medium \\
\hline
\end{tabular}




\begin{tabular}{|c|c|c|c|c|c|c|c|}
\hline Amenazas / Threats & Balsillas & $\begin{array}{l}\text { Punta } \\
\text { Gorda }\end{array}$ & $\begin{array}{l}\text { Punta } \\
\text { Seca }\end{array}$ & $\begin{array}{l}\text { Boca Ana } \\
\text { Gómez }\end{array}$ & Chichimán & Subtotal & $\begin{array}{c}\text { Grado de amenaza / } \\
\text { Threat level }\end{array}$ \\
\hline $\begin{array}{l}\text { Contaminación lumínica / } \\
\text { Light pollution }\end{array}$ & 0 & 0 & 3 & 1 & 0 & 4 & Medio / Medium \\
\hline $\begin{array}{l}\text { Saqueo de nidos / } \\
\text { Poaching of eggs }\end{array}$ & 0 & 1 & 1 & 0 & 3 & 5 & Medio / Medium \\
\hline $\begin{array}{l}\text { Depredación por animales / } \\
\text { Depredation by animals }\end{array}$ & 1 & 1 & 3 & 3 & 2 & 10 & Alto / High \\
\hline $\begin{array}{l}\text { Contaminación por residuos } \\
\text { sólidos / Solid waste pollution }\end{array}$ & 0 & 1 & 0 & 3 & 3 & 7 & Alto / High \\
\hline $\begin{array}{l}\text { Obstáculos en playa / } \\
\text { Obstacles on beaches }\end{array}$ & 0 & 1 & 0 & 3 & 3 & 7 & Alto / High \\
\hline $\begin{array}{l}\text { Tránsito de ganado / } \\
\text { Livestock transit }\end{array}$ & 0 & 0 & 0 & 0 & 1 & 1 & Bajo/ / Low \\
\hline $\begin{array}{l}\text { Tránsito de personas / } \\
\text { Human transit }\end{array}$ & 2 & 2 & 2 & 0 & 2 & 8 & Alto / High \\
\hline Pesca incidental / Incidental catch & 1 & 0 & 1 & 1 & 3 & 6 & Medio / Medium \\
\hline $\begin{array}{l}\text { Pesca destructiva / } \\
\text { Destructive fishing }\end{array}$ & 2 & 2 & 0 & 0 & 3 & 7 & Alto / High \\
\hline $\begin{array}{l}\text { Tránsito marítimo / } \\
\text { Marine transport }\end{array}$ & 1 & 1 & 1 & 0 & 2 & 5 & Medio / Medium \\
\hline Subtotal & 10 & 10 & 19 & 11 & 30 & & \\
\hline $\begin{array}{l}\text { Grado de Amenaza / } \\
\text { Level of Threat }\end{array}$ & $\begin{array}{l}\text { Bajo / } \\
\text { Low }\end{array}$ & $\begin{array}{l}\text { Bajo / } \\
\text { Low }\end{array}$ & $\begin{array}{l}\text { Medio / } \\
\text { Medium }\end{array}$ & $\begin{array}{l}\text { Medio / } \\
\text { Medium }\end{array}$ & Alto / High & & \\
\hline
\end{tabular}

\section{DISCUSIÓN}

El monitoreo sistemático diurno y nocturno de las zonas de anidación de la tortuga carey es indispensable para conocer el estado actual de la especie en el país. Por lo cual, al igual que Barrientos et al. (2015), se recomienda dar continuidad al monitoreo estandarizado de la especie en Rincón del Mar, para determinar la tendencia de la población reproductiva en el área. La frecuencia, tipo y esfuerzo en el monitoreo determinan la efectividad para identificar la situación actual de la especie y los esfuerzos donde los monitoreos deben de ser enfocados. Por lo tanto, en lugares de difícil acceso o con anidación esporádica como Rincón del Mar, los investigadores locales con base comunitaria son indispensables para llevar a cabo este monitoreo, que llevó al registro de seis nidadas durante los meses de mayor anidación.

El tamaño de la nidada es similar a lo reportado por otros estudios con la tortuga carey en el Caribe y Pacífico (Tabla 6). El éxito de eclosión (78,2 \%) en Rincón del

\section{DISCUSSION}

Systematic daytime and nocturnal monitoring of the nesting sites of the hawksbill turtle is indispensable if the current status of the species in Colombia is to be understood. Therefore, like Barrientos et al. (2015), the authors recommend continuing with a standardized monitoring process of the species in Rincón del Mar, in order to determine the trends of the reproductive population in the area. The frequency and kind of monitoring, and the efforts put into the monitoring process will decide its effectiveness in identifying the current situation of the species and the areas on which future monitoring should focus. Therefore, in places such as Rincón del Mar, which are difficult to reach and where nesting is sporadic, local researchers with community roots are indispensable to this kind of monitoring, which recorded the laying of six clutches during the principal nesting months.

The size of the clutches is similar to those reported in other studies of the hawksbill turtle in the Caribbean and 
Mar es superior a lo registrado por Chacón et al. (2004), Colombia Marina (2007), Hancock (2008), Gerhartz-Muro et al. (2019) y Liles et al. (2019), incluyendo una previa aproximación a la especie en el área por Caraballo et al. (2008) (Tabla 6), lo que sugiere que las playas de Rincón del Mar presentan características óptimas para que las nidadas tengan un desarrollo embrionario completo y una alta tasa de supervivencia de las nidadas. Algunos factores determinantes para la supervivencia de las nidadas son la humedad, la temperatura, el tipo de arena, la pendiente y extensión de la playa (Bolongaro et al., 2010; Piedra-Castro y Morales-Cerdas, 2015). Es probable que la presencia de la vegetación nativa de las playas de Rincón del Mar (Tabla 3) esté brindando las características ideales de una zona de anidación, que para esta especie son indispensables al desovar su nidada y mantener las condiciones microclimáticas estables.

Tabla 6. Resumen del total de huevos en nidadas (huevos), éxito de eclosión (\%) (promedio \pm DE y n) de E. imbricata de Rincón del Mar, Sucre y otros estudios realizados en el Caribe y Pacífico. n.r. $=$ no registrado.
Pacific coastal regions of the Americas (Table 6). Hatching success $(78.2 \%)$ is higher in Rincón del Mar than the levels reported by Chacón et al. (2004), Colombia Marina (2007), Hancock (2008), Gerhartz-Muro et al. (2019) and Liles et al. (2019), including a prior estimation for the same area by Caraballo et al. (2008) (Table 6). This finding suggests that the beaches of Rincón del Mar provide optimal conditions for complete embryonic development to occur in nests and for high rates of survival. Among the determining factors for the survival of clutches are humidity, temperature, sand type and the slope and extension of beaches (Bolongaro et al., 2010; Piedra-Castro and Morales-Cerdas, 2015). It is probable that the presence of native vegetation on the beaches of Rincón del Mar (Table 3) ensures ideal conditions in nesting zones, which are vital to hatching success of this species, as they help maintain stable microclimatic conditions.

Table 6. Summary of average values of clutch size (eggs), hatching success (\%) (mean $\pm \mathrm{SD}$ and $\mathrm{n}$ ) of E. imbricata in Rincón del Mar, compared to other studies on the Caribbean and Pacific coastal regions. n.r. = not registered.

\begin{tabular}{|c|c|c|c|}
\hline $\begin{array}{c}\text { Promedio del Total de huevos en nidada / } \\
\text { Average total number of eggs in clutch (h) }\end{array}$ & $\begin{array}{c}\text { Éxito de eclosión / } \\
\text { Hatching success (\%) }\end{array}$ & $\begin{array}{c}\text { Lugar / Location } \\
\text { Referencia / Reference }\end{array}$ & Chacón et al. (2004) \\
\hline n.r. & $76(14)$ & Limón, Costa Rica & Colombia Marina (2007) \\
\hline $163.9 \pm 16.7(37)$ & $61.3(1)$ & Limón, Costa Rica & Hancock (2008) \\
\hline $132.8 \pm 44.6(4)$ & $77.4 \pm 31.1(6)$ & Sucre, Colombia & Caraballo et al. (2008) \\
\hline $147.8 \pm 52.6(6)$ & $0(4)$ & Pinar del Río, Cuba & Gerhartz-Muro et al. (2018) \\
\hline $160.2 \pm 37.8(2031)$ & $33.6 \pm 25.1(6)$ & Bahía de Jiquilisco, El Salvador, y & Liles et al. (2019) \\
\hline $152.2 \pm 15.5(5)$ & $56.8 \pm 30.2(2031)$ & Sucre, Colombia & Este estudio \\
\hline
\end{tabular}

Por otra parte, las medidas biométricas de LRC de $4,0 \pm 0,4 \mathrm{~cm}$, de ARC de 2,9 $\pm 0,2 \mathrm{~cm}$ y peso total de 15,4 $\pm 0,5 \mathrm{~g}$ en Rincón del Mar son similares a lo registrado en otros estudios realizados en el Caribe y Pacífico (Kaufmann, 1975; Chacón et al., 2004; Joya y Molina, 2006; Hancock, 2008; Liles et al., 2019) (Tabla 4).

La educación ambiental, la participación de la comunidad de Rincón del Mar y el trabajo interinstitucional realizado fueron indispensables para la protección de los nidos in situ hasta el momento de la eclosión. El fortalecimiento comunitario sobre la importancia de la tortuga carey incentivó a la comunidad en apoyar
On the other hand, the biometric measures in Rincón del Mar (LRC of $4.0 \pm 0.4 \mathrm{~cm}$, ARC of $2.9 \pm 0.2 \mathrm{~cm}$ and total weight of $15.4 \pm 0.5 \mathrm{~g}$ ) are similar to those of other studies conducted in Pacific and Caribbean coastal regions (Kaufmann, 1975; Chacón et al., 2004; Joya and Molina, 2006; Hancock, 2008; Liles et al., 2019) (Table 4).

Environmental education, the participation of the Rincón del Mar community and the interinstitutional activities carried out were indispensable to the protection of the nests in situ up to the point of hatching. Community strengthening activities focused on the importance of the hawksbill turtle provided incentives to inhabitants to support 
acciones de conservación para la especie. La inclusión y la participación comunitaria son un paso importante hacia la conservación de las tortugas marinas, siendo fundamentales para el monitoreo, teniendo en cuenta la baja frecuencia de nidos en el sector y su significancia por la conectividad que representa con otras zonas marino costeras aledañas. Así como en otras partes del mundo, en donde los proyectos participativos en un largo periodo de tiempo han llevado a estabilizar o incluso incrementar las poblaciones de tortugas marinas (Godley et al., 2020), se espera que la continuidad de estos procesos logre afianzar el sentido de pertenencia por parte de la comunidad y un trabajo mancomunado con grupos y/o entidades protectoras del medio ambiente.

Finalmente, en cuanto a las amenazas para las zonas de anidación en Rincón del Mar, las más severas incluyen la erosión costera, la depredación de los nidos y la contaminación por desechos sólidos, las cuales también fueron identificadas previamente por Caraballo et al. (2008) y por Rincón-Díaz y Rodríguez-Zárate (2004) en el archipiélago de San Bernardo. En el corregimiento de Rincón del Mar la erosión costera presenta una vulnerabilidad de media a alta (Corporación Ecoversa , 2018), principalmente en el sector norte hacia Chichimán, a pesar de tener las playas más extensas y con cobertura vegetal consolidada (Caraballo et al., 2008). El mayor grado de amenaza encontrado en Chichimán es ocasionado probablemente por una acelerada pérdida de barreras naturales de la línea costera, como manglares, pastos marinos y formaciones coralinas, sumada a la limitada intervención de las autoridades por el contexto social que aún persiste en la región . De igual manera, en el sector de Punta Gorda, debido a la erosión se necesitaron sacos de arena para evitar que dos de los nidos fueran lavados por el mar.

Respecto a la depredación de los nidos, la presencia de animales domésticos como los perros y cerdos en las playas de anidación es alarmante, ya que pone en riesgo las colonias anidantes de tortugas marinas (Kontos 1985, 1987, 1988; Richardson, 1990; Suganuma, 2005; Andrews et al., 2006; Meylan et al., 2006; Whytlaw, 2013; Engeman et. al. 2016, 2019). Por otro lado, el consumo y comercialización de nidadas de tortugas marinas también sigue siendo una práctica común, como en otros lugares del país, incluso en áreas bajo alguna figura de protección (Rincón-Díaz y Rodríguez-Zárate, 2004; Barrientos et al., 2013 , 2014, 2015, 2020; Moreno-Munar et al., 2014 ). Un ejemplo de ésto es lo ocurrido durante este estudio, en donde, a pesar de haberse instalado encierros demarcados sobre los nidos, actions to protect the species. Community inclusion and participation are an important step towards the conservation of marine turtles, as the local population is fundamental to monitoring, bearing in mind the infrequency of nests in the sector and their importance in the interconnections with other neighboring marine coastal areas. Thus, as in other parts of the world, where long-term participatory processes have stabilized or even increased marine turtle populations (Godley et al., 2020), it is to be hoped that the continuation of these actions will consolidate the sense of ownership of the community and coordination with environmental protection groups and/or agencies.

Finally, of the threats to the nesting zones in Rincón del Mar, the most serious include coastal erosion, the depredation of nests and solid waste pollution, all of which had previously been identified by Caraballo et al. (2008) and by Rincón-Díaz and Rodríguez-Zárate (2004) for the archipelago of San Bernardo. In the village of Rincón del Mar, coastal erosion represents a medium to high threat (Corporación Ecoversa, 2018), principally in the northern sector towards Chichimán, despite the fact that it is here that the most extensive beaches are found and the vegetation is most consolidated (Caraballo et al., 2008). The greatest degree of threat in Chichimán is probably the result of the accelerated loss of natural barriers along the coastline, such as mangroves, marine grasses and coral formations, alongside the limited role of the authorities, due to the public order situation that still characterizes the area. Similarly, because of erosion in the sector of Punta Gorda, sandbags were required to protect the nests from being washed away by the sea.

In terms of the depredation of nests, the presence on the nesting beaches of domestic animals such as dogs and pigs is alarming, as they represent a risk to laying marine turtle colonies (Kontos 1985, 1987, 1988; Richardson, 1990; Suganuma, 2005; Andrews et al., 2006; Meylan et al., 2006; Whytlaw, 2013; Engeman et. al. 2016, 2019). On the other hand, the consumption and commercialization of marine turtle clutches also remains a common practice, as it is also in other parts of the country, including in areas that benefit from some form of protection (Rincón-Díaz and Rodríguez-Zárate, 2004; Barrientos et al., 2013, 2014, 2015, 2020; Moreno-Munar et al., 2014). An example of this occurred during the period reported here, when, despite the fencing off and labeling of nests, one was raided. This demonstrates the high degree of exposure to depredation by outside species or human beings and the need to continue 
uno de éstos fue saqueado. Lo que demuestra el alto grado de exposición a la depredación por especies foráneas o humanos y la necesidad de continuar con labores de monitoreo, protección y sensibilización en el área.

La captura de adultos para consumo y comercialización del caparazón para el tráfico de carey es también una amenaza para la especie en Rincón del Mar (Caraballo et al., 2008), como en el resto de la costa Caribe de Colombia, siendo el segundo país con mayor captura de tortuga carey en el mundo, con un estimado de más de 600 tortugas por año (Campbell, 2014; Humber et al., 2014; Barrientos et al., 2015, 2020; Ramírez-Gallego y Barrientos-Muñoz, 2020a, 2021). En el caso de Sucre, la isla de Boquerón se ha identificado como el centro de mayor captura de tortugas verde y carey de manera directa empleando redes tortugueras (Rincón-Díaz y RodríguezZárate, 2004) y en Sabanetica por captura incidental en redes agalleras. Para combatir esto, Carsucre (2017) en su jurisdicción ha decomisado $6600 \mathrm{~m}$ de redes tortugueras en 2015-2017 y ha propuesto la declaratoria del DRMI Sabanetica, Chichimán, Rincón del Mar y Berrugas, cómo una figura de protección del área para la conservación la tortuga carey.

La contaminación por residuos sólidos, a su vez, es de gran preocupación, ya que las playas tenían una alta acumulación de plásticos. Lo que puede afectar las propiedades de los nidos, como la temperatura y la permeabilidad, e impactar de manera directa a las hembras anidantes y las crías por ahogamiento u obstaculización (Nelms et al., 2015).

Otros factores identificados en campo que afectan de manera indirecta y que requieren atención incluyen el uso de artes de pesca destructivas como chinchorro playero y pesca con dinamita sobre áreas coralinas, la pérdida de hábitat por erosión costera, la tala ilegal del manglar y el desarrollo costero. Estos factores actúan de manera sinérgica como agentes transformadores de los ecosistemas, afectando los procesos de ovoposición por la disminución del espacio disponible e influir en cambios en las características del entorno (composición de la arena, sombreo por la vegetación) que podrían incidir en la disminución del éxito de eclosión y de emergencia de las nidadas. with monitoring, protection and awareness-raising activities in the area.

The capture of adult turtles for human consumption and the commercialization of their shells in the context of tortoiseshell smuggling also represent a threat to the species in Rincón del Mar (Caraballo et al., 2008), as it does in the rest of the Caribbean coast of Colombia: the country where the second highest number of hawksbill turtles in the world -an estimated 600- is captured annually (Campbell, 2014; Humber et al., 2014; Barrientos et al., 2015, 2020; Ramírez-Gallego and Barrientos-Muñoz, 2020a, 2021). In the case of the department of Sucre, the Isla de Boquerón has been identified as a major center for the capture of green and hawksbill turtles using the direct method of turtle nets (Rincón-Díaz and Rodríguez-Zárate, 2004), while in Sabanetica they are an incidental catch of fishing using gillnets. In response to this, Carsucre (2017) decommissioned $6600 \mathrm{~m}$ of turtle nets between 2015 and 2017 in the area under its jurisdiction and has proposed the establishment of the Regional Integrated Management District of Sabanetica, Chichimán, Rincón del Mar and Berrugas as a mechanism to protect the area for the conservation of the hawksbill turtle.

Solid waste pollution is also a major concern, as a large amount of plastic is found on the beaches. This has a potential effect on different properties of the nests, including temperature and permeability and has a direct impact on laying females and hatchlings by causing suffocation or presenting physical obstacles (Nelms et al., 2015).

Other factors identified in the field with indirect effects that require attention include the employment of destructive fishing techniques such as chinchorros playeros (beach seines, or cast nets) and dynamite in coral reef areas, habitat loss caused by coastal erosion, illegal felling of mangroves and coastal development, All of these act synergically to transform the ecosystems of the area, affecting the laying process by reducing available space. They also produce changes in the characteristics of the environment (sand composition, provision of shade by vegetation) that might reduce hatching and emergence success. 


\section{CONCLUSIONES}

Las playas continentales del Caribe colombiano identificadas para la anidación de la tortuga carey son pocas y con anidación esporádica. En Rincón del Mar y áreas circundantes la abundancia de nidos es reducida, sin embargo E. imbricata continúa dando uso a estas playas para el desove de sus nidadas a pesar de las amenazas locales. Por lo cual, siendo la tercera zona con mayor abundancia para la anidación de la tortuga carey en playas continentales, es indispensable fortalecer procesos de protección precisando mecanismos con esfuerzos de conservación dirigidos desde la participación comunitaria y el trabajo interinstitucional e intersectorial.

Por otra parte, las condiciones geomorfológicas de la playa y la composición vegetal pueden estar influyendo para que se mantenga la filopatria de la especie a la zona, permitiéndoles a las hembras realizar una óptima selección del sitio de postura dejar lo nidos in situ. Tal como se como se comprobó en este estudio, con un alto éxito reproductivo de las nidadas. No obstante, se ha observado que algunas playas están siendo modificadas, no sólo por variables ambientales, sino por la influencia antrópica.

Estas playas son un lugar clave para proteger una de las etapas más críticas en el ciclo de vida de $E$. imbricata, beneficiando así la conservación de la especie en esta amplia región en donde existe e incluso sobrelapa un mosaico de áreas marinas y costeras protegidas, como lo son el Área Marina Protegida Archipiélago del Rosario y San Bernardo, el Parque Nacional Natural Los Corales del Rosario y de San Bernardo y, en proceso de declaratoria, el DRMI Sabanetica, Chichimán, Rincón del Mar y Berrugas. Por lo tanto, es urgente establecer un plan de manejo interinstitucional e intersectorial para su protección.

En el plan de manejo de la especie deben incluirse recursos económicos permanentes para un monitoreo a largo plazo, saturación de marcaje y transmisores satelitales que nos permitan conocer sus rutas migratorias, uso y conectividad del área, además de un programa de educación ambiental que sea transversal a toda la comunidad y la generación de un programa de alternativas económicas locales sostenibles que ayude a eliminar el uso de subproductos de la tortuga carey.

\section{CONCLUSIONS}

The beaches of Colombia's Caribbean mainland on which the hawksbill turtle nests are few and nesting is sporadic. In Rincón del Mar and surrounding areas nests are not abundant. However, despite the local threats, E. imbricata continues using these beaches to lay its eggs. Consequently, as the zone hosts the third highest number of continental beaches on which hawksbill turtles nest in the country, it is of vital importance to strengthen protection processes by defining conservation mechanisms based on community participation, alongside interinstitutional and intersectoral approaches.

On the other hand, the geomorphological conditions of the beaches and the composition of their vegetation might be exerting an influence that helps maintain the philopatry of the species, permitting the females, also, to select the ideal spot to lay their eggs and to preserve their nesting sites in situ, with a high degree of reproductive success, as has been shown in this study. However, it has been observed that some beaches are being modified, not only in terms of their environmental variables, but also as a result of anthropic influences.

These beaches are key sites for the protection of the most critical stages in the life cycle of E. imbricata; they favor the conservation of the species in this extensive region, which consists of a mosaic of sometimes overlapping protected marine and coastal areas, including the Área Marina Protegida Archipiélago del Rosario y San Bernardo, the Parque Nacional Natural Los Corales del Rosario y de San Bernardo and the DRMI Sabanetica, Chichimán, Rincón del Mar y Berrugas (currently in the process of being established). It is therefore urgently important to establish an interinstitutional and intersectoral protection plan.

This species management plan should include the provision of permanent financial resources to ensure long-term monitoring, the saturation tagging and satellite transmission required to understand their use of habitats and the level of connectivity between these areas, an environmental education program that reaches the entire community and the development of a program offering local economic alternatives that would help eliminate the exploitation of hawksbill turtle byproducts. 


\section{AGRADECIMIENTOS}

El proyecto no hubiese sido posible sin el trabajo de los Guardianes de las Tortugas de Rincón del Mar: Nehemías Tapia Robles, Johonis Tapia Robles, Arnold Díaz Blanco, José Pedro Guerrero Contreras, Gregorio Contreras Meza, Juan Silgado Flórez, Jorge Tapia, Denerys Blanco Arrieta, Luis Eduardo Zúñiga Contreras, Jhon Enrique Ramos Ocón, Jeisson Ramos Ocón y Johanis Patricia Tapia Robles, su colaboración, compromiso, toma de datos y protección de las nidadas fue crucial para el proyecto. A la Corporación Autónoma Regional de Sucre, en cabeza de su director Johnny Avendaño Estrada y a Jesús Romaní Lobo por su apoyo en los monitoreos diurnos y nocturnos durante 2018 . Un especial agradecimiento a todos los niños de Rincón del Mar, por su compromiso con la conservación de las tortugas marinas, principalmente a la Institución Educativa Rincón Del Mar, con el grupo ambiental Guardianes Apasionados por las Tortugas Marinas liderado por la Profesora Delcy Lares Ramos, y a los Titanes Ecológicos. Al condominio Balsillas y su administrador Martín Álvarez, la Asociación Colombian Reef (Alberto González y Julie Lobbestael), Dos Aguas Lodge (Dania Bianuni y Daniele Bertolotti), Canto de Caracola (Angela Olarte) y Casita B lanca (familia Tapia Robles) por el apoyo en hospedaje y logístico durante el proyecto. Los autores agradecen especialmente a la familia Tapia Robles que por muchos años ha protegido a la tortuga carey en Rincón del Mar. Financiado en el marco del proyecto "Manejo integrado de la UAC estuarina río Sinú-Golfo de Morrosquillo" de Carsucre y la Fundación Tortugas del Mar.

\section{ACKNOWLEDGEMENTS}

This project would not have been possible without the work of the Guardianes de las Tortugas de Rincón del Mar: Nehemías Tapia Robles, Johonis Tapia Robles, Arnold Díaz Blanco, José Pedro Guerrero Contreras, Gregorio Contreras Meza, Juan Silgado Flórez, Jorge Tapia, Denerys Blanco Arrieta, Luis Eduardo Zúñiga Contreras, Jhon Enrique Ramos Ocón, Jeisson Ramos Ocón and Johanis Patricia Tapia Robles. Their collaboration, commitment, data collection efforts and their work to protect the clutches were crucial to the project. The authors thank the Corporación Autónoma Regional de Sucre, represented by its director Johnny Avendaño Estrada and Jesús Romaní Lobo for their assistance with the day-time and nocturnal patrols in 2018. Particular thanks go to the children of Rincón del Mar, for their commitment to the protection of marine turtles, and in particular to the Institución Educativa Rincón Del Mar and the environmental group Guardianes Apasionados por las Tortugas Marinas, led by the teacher Delcy Lares Ramos, and to the Titanes Ecológicos. To the Balsillas condominium and its administrator Martín Álvarez, la Asociación Colombian Reef (Alberto González and Julie Lobbestael), the Dos Aguas Lodge (Dania Bianuni and Daniele Bertolotti), the Canto de Caracola (Angela Olarte) and the Casita Blanca (the Tapia Robles family) for their accommodation and logistical support during the project. The authors express particular gratitude to the Tapia Robles family which has, for many years, protected the hawksbill turtles of Rincón del Mar. This project received financial support from Carsucre and Fundación Tortugas del Mar program, Manejo integrado de la UAC estuarina río Sinú-Golfo de Morrosquillo.

\section{BIBLIOGRAFÍA / LITERATURE CITED}

Andrews, H.V., S. Krishnan and P. Biswas. 2006. Distribution and status of marine turtles in the Andaman and Nicobar Islands: $33-57$ in Shanker, K. and B. Choudhury (E ds. ) Marine turtles of the Indian subcontinent. Universities, Hyderabad, India.

Arcos, M., A. Barrero, G. Guarín y P. Quintero. 2002. Establecimiento y comparaciones de características estructurales de puntos focales de anidación de tortugas marinas ya establecidos en el sector de Arrecifes Parque Nacional Natural Tayrona, Caribe colombiano. Seminario de investigación, Univ. Jorge Tadeo Lozano, Santa Marta. 54 p.

Barrientos-Muñoz, K.G., C. Ramírez-Gallego and L. Rivas. 2013. First report of nesting of the Black Sea turtle (Chelonia mydas) on the north Pacific coast of Colombia. Mar. Turtle Newslet., 138: 19-21. http://www.seaturtle.org/mtn/archives/mtn138/mtn138-7.shtml

Barrientos-Muñoz, K.G., C. Ramírez-Gallego and V. Páez. 2014. Nesting ecology of olive Ridley (Lepidochelys olivacea) (Cheloniidae) at El Valle Beach, northern Pacific, Colombia. Acta Biol. Col., 19(3): 437-445: https://dx.doi.org/10.15446/abc.v19n3.42457

Barrientos-Muñoz, K., C. Ramírez-Gallego y V.P. Páez. 2015. Eretmochelys imbricata (Linnaeus, 1766): 127-131. En: Morales-Betancourt, M.A., C.A. Lasso, V.P. Páez y B.C. Bock (Eds.). Libro rojo de reptiles de Colombia. Inst. Invest. Rec. Biol. Alexander von Humboldt (IAvH), Univ. Antioquia, Bogotá, D.C. 258 p. 
Barrientos-Muñoz, R. A. Álvarez-Rodrí guez, W. Álzate-Montoya, J. Arends, J. Bernal-Gutiérrez' S. Bessudo, J. P. Caldas, E. Córdoba-Borja, M.C Diazgranados, J. Herrera, K. Iguarán Quijada, A. Jáuregui-Romero, F. Ladino, H. Marimó n, V. Márquez, A. Carlos Mata, J. David Miranda, J. Moreno-Sotomayor, V. P. Páez, L. Payan, M.F Pereira-Gutiérrez, D. Ramírez-Acosta, E. Ramírez-Gáfaro, N. Restrepo-Garzó n, E.L Robledo-Leal, J. Tapia-Robles, N.J Tapia-Robles, S. Valencia-González, M. Valencia, M. X. Zorrilla - Arroyave y C. Ramírez-Gallego. 2020. Eretmochelys imbricata (Linnaeus 1766). En revisión. Catálogo de anfibios y r eptiles de Colombia. Asociación Colombiana de Herpetología.

Bolongaro, A., Z. Márquez, V. Torres y A. García. 2010. Vulnerabilidad de sitios de anidación de tortugas marinas por efectos de erosión costera en el estado de Campeche: 73-96. En: Botello, A.V., S. Villanueva-Fragoso, J. Gutiérrez y J.L. Rojas Galaviz (Eds.) Vulnerabilidad de las zonas costeras mexicanas ante el cambio climático. Semarnat-INE, UNAM-ICMyL. Univ. Autón. Campeche, Campeche. 514 p.

Campbell, C.L. 2014. Estado de c onservación de la tortuga c arey en las regiones del Gran Caribe, Atlántico Occidental y Pacífico Oriental. Secretaría Pro Tempore CIT, Virginia . $76 \mathrm{p}$

Caraballo-Pérez, M., M. Fernández-Pérez y A. Zamora-Guzmán . 2008. Caracterización de zonas potenciales de anidación de Eretmochelys imbricata y búsqueda de su anidamiento en el sector punta Seca - Rincón del Mar, departamento de Sucre. Sincelejo: Conservación Internacional - Colombia, Fundación Omacha, CARSUCRE, Universidad de Sucre. 24 p.

Cardique, Carsucre, Codechocó, Coralina, orpamag, Corpoguajira, Corponariño, Corpourabá, CRA, CRC, CVC, CVS, Invemar, MADS y PNN. 2016. Plan de Acción del Subsistema de Áreas Marinas Protegidas - SAMP 2016-2023: Lineamientos para su consolidación en el marco de los Subsistemas Regionales de Áreas Protegidas del Pacífico y del Caribe. Zamora-Bornachera, A.P. (Ed.) Proyecto COL75241, PIMS \# 3997, Invemar, MADS, GEF y PNUD. Serie Publ. Gen. Invemar 85, . 60 p.

Carsucre . 2017. Informe de actividades de control y vigilancia flora y fauna enero-junio. Bol. Amb. 1. Sincelejo, Sucre.

Ceballos-Fonseca, C. 2004. Distribución de playas de anidación y áreas de alimentación de tortugas marinas y sus amenazas en el Caribe colombiano. Bol. Invest. Mar. Cost., 33: 79-99 doi: 10.25268/bimc.invemar.2004.33.0.249.

Chacón, D., A. Morales y E.V. Passapera. 2004. Informe de la anidación de la tortuga de carey Eretmochelys imbricata en el Parque Nacional Cahuita, Limón, Costa Rica. Proyecto para la Conservación de las Tortugas Marinas en el Caribe Sur. Asoc. ANAI, Talamanca, Costa Rica.

Chacón, D., J. Sánchez, J.J. Calvo y J. Ash. 2007. Manual para el manejo y la conservación de las tortugas marinas en Costa Rica; con énfasis en la operación de proyectos en la playa y viveros. Asociación ANAI, SINAC, MINAE). Gobierno de Costa Rica, San José. 103 p.

Colombia Marina. 2007. Project: Strengthening the conservation process of nesting sea turtles in Tayrona National Park - Colombian Caribbean. Season 2007. Final r eport. Santa Marta . 16 p.

Cusado-Zapa, C.A. y A.M. González -Pérez. 2010. Análisis fitosociológico del ecosistema de manglar del parque natural boca Guacamayas, departamento de Sucre. Sincelejo-Sucre. Trabajo de grado Biología, Univ. Sucre. Sincelejo, Colombia.

Duque, D., B. Medrano y R. Franke. 2011. Avances de un proceso participativo para generar línea base para monitoreo de tortugas marinas en el área protegida y su zona de influencia. Capítulo 26: 351-363 . En: Zarza-González, E. (Ed.). Entorno Ambiental del Parque Natural Corales del Rosario. Parques Nacionales Naturales de Colombia. Quito Publicidad, Cartagena de Indias. 416 p.

Eckert, K.L. and A.E. Eckert. 2019. An atlas of sea turtle nesting habitat for the wider Caribbean region. Revised Edition. WIDECAST Tech. . Rep., 19. Godfrey, USA. 232 p.

Ecoversa . 2018. Estudio técnico para la declaratoria de punta San Bernardo y Chichimán - Rincón del Mar en el municipio de San Onofre (Sucre) como Área Protegida Regional y formulación del plan de manejo, en el marco del Proyecto GCP/COL/041/GFF. Medellín . 120 p.

Engeman, R.M., D. Addison and J.C. Griffin. 2016. Defending against disparate sea turtle nest predators: benefits to nesting success from eradicating invasive feral swine and caging nests from raccoons. Oryx, 50(2): 289-295.

Engeman, R.M., R.W. Byrd., J. Dozier., M.A. McAlister., J.O. Edens., E.M. Kierepka., T.J. Smyser. and N. Myers. 2019. Feral swine harming insular sea turtle reproduction: The origin, impacts, behavior and elimination of an invasive species. Acta Oecol. , 99: 103442 https://doi.org/10.1016/j. actao.2019.103442

Gerhartz-Muro, J.L., J. Azanza., F. Moncada, A. Gerhartz-Abraham, L. Espinosa, Y. Forneiro, and D. Chacón. 2019. Su n and i ncubation temperatures in a sea turtle nesting beach at the cayos de San Felipe National Park, Pinar del Río, Cuba, during the 2012-2013 season. Rev. Invest. Mar. , 38(2): 100-116.

Godley, B. J., A.C. Broderick, L.P. Colman, A. Formia, M.H. Godfrey, M. Hamann and A.F. Rees. 2020. Reflections on sea turtle conservation. Oryx, 54(3): 287-289. https://doi.org/10.1017/S0030605320000162

Hancock, J. 2008. Monitoreo de la anidación de la t ortuga carey (Eretmochelys imbricata) y de la tortuga verde (Chelonia mydas) en el Parque Nacional Cahuita, Caribe sur, Costa Rica. Inf. Activ. . WIDECAST, Costa Rica. 33 p.

Humber, F., B.J. Godley and A.C. Broderick. 2014. So excellent a fishe: a global overview of legal marine turtle fisheries. Div. Distrib. , 20(5): 579-590. doi : 10.1111/ddi.12183.

INVEMAR-CVS. 2012. Lineamientos de manejo integrado de la Unidad Ambiental Costera Estuarina río Sinú -golfo de Morrosquillo. Hernández-Ortiz, M., A. P. Zamora-Bornachera y A. López-Rodríguez (Eds.). Ser. Doc. G en. Invemar 53.79 p. 
Joya, A. M. y M.P. Molina. 2006. Levante de neonatos de t ortuga carey, Eretmochelys imbricata (L inneaus, 1766) mediante la implementación de dos tipos de dietas en el acuario Mundo Marino, Santa Marta. Trabajo grado Biol. Mar., . Universidad Jorge Tadeo Lozano. Santa Marta . 133 p.

Kaufmann, R. 1967. Wachtstumsraten in Gefangenschaft gehaltener Meersschildkröten. Mitteilun. Inst. Colombo-Alemán Invest. Cient. Punta de Betín, 1: $65-72$.

Kaufmann, R. 1975. Observaciones sobre el crecimiento de tortugas marinas en cautividad. Caldasia, 139-150. doi: 10.15446/caldasia.

Kontos, A.R. 1985. Sea turtle research report, 1985, Mona Island, Puerto Rico. An. Rep. ort U.S. Fish Wildl. Serv. . 35 p.

Kontos, A.R. 1987. 1986 annual summary: estimation of sea turtle abundance and nesting success of Mona Island, Puerto Rico. An. Rep. ort U.S. Fish Wildl. Serv. $22 \mathrm{p}$.

Kontos, A.R. 1988. 1987 annual summary: estimation of sea turtle abundance, Mona Island, Puerto Rico. An. Rep. ort U.S. Fish Wildl. Serv. . 24 p.

Liles, M.J., T.R. Peterson, J.A. Seminoff, A.R. Gaos, E. Altamirano, A.V. Henríquez, V. Gadea, S. Chavarria, J. Urteaga, B.P. Wallace and M.J. Peterson. 2019. Potential limitations of behavioral plasticity and the role of egg relocation in climate change mitigation for a thermally sensitive endangered species. Ecol. Evol. , 9(4): 1603-1622. doi: 10.1002/ece3.4774

McCormick, C.C. 1997. Porque ellas también tienen derecho a seguir dejando huella. Diagnóstico actual de las tortugas marinas del archipiélago de San Andrés, Providencia y Santa Catalina, Fase II.CORALINA, San Andrés. 67 p.

McCormick, C.C. 1998. Diagnóstico actual de las tortugas marinas del archipiélago de San Andrés, Providencia y Santa Catalina. Coralina, San Andrés. $41 \mathrm{p}$.

Meylan, A.B. 1999. Status of the hawksbill turtle (Eretmochelys imbricata) in the Caribbean r egion. Chel. Cons. Biol., 3(2): $177-184$.

Meylan, A.B. and M. Donelly. 1999. Status justification for listing the hawksbill turtle (Eretmochelys imbricata) as Critically Endangered on the 1996 IUCN Red List of Threatened Animals. Chel. Cons. Biol., 3(2): 200-224.

Meylan, A.B., I. Castillo, N. Decastro, C. Ordó ñez, S. Troëng, A. Ruiz and P.A. Meylan. 2006. Bastimentos Island National Marine Park and Playa Chiriqui: protected areas vital to the recovery of the hawksbill turtle (Eretmochelys imbricata) in Caribbean Panama: 145- 146. In: Frick, M., A. Panagopoulou, A.F. Rees and K. Williams (compilers). Abstracts Twenty-sixth $26^{\text {th }}$ An. Symp. Sea Turtle Biol. Cons . Internat. Sea Turtle Society. Athens .

Miller, J. 1999. Determining c lutch size and hatching success: 124-129. I n: Eckert K., K.A. Bjorndal, F.A. Abreu-Grobois and M. Donnelly (Eds.) Research and management techniques for the conservation of sea turtles. IUCN/SSC Mar. Turt. Spec. Group Publ. 4. Washington, D.C. 248 p.

Moncada, F., C. Ramírez-Gallego, J. Camero, M. Gonz ález, G. Nodarse and K.G. Barrientos-Muñoz. 2019. A juvenile loggerhead turtle (Caretta caretta) tagged in Cuba is recaptured in Colombian waters. Mar. Turtle Newslet., 156: 30-32. http://www.seaturtle.org/mtn/archives $/ \mathrm{mtn} 156 / \mathrm{mtn} 156-9 . \operatorname{shtml}$

Moreno-Munar, Á. A., S.C. Ospina-Sánchez, A.G. Jáuregui-Romero y R. Álvarez-León. 2014. Monitoramiento de poblaciones de tortugas marinas en los sectores de Arrecifes y Cañaveral, Parque Nacional Natural Tayrona, Colombia. Arq. Cien. Mar. Fortaleza, 47: 19-30. Available online at: http:// www. repositorio.ufc.br/bitstream/riufc/28712/1/2014_art_aammunar.pdf

Mortimer, J.A. and M. Donnelly . 2008. Eretmochelys imbricata. IUCN Red List Threatened Species 2008. Version 2014.3.1 www.iucnredlist.org. $06 / 12 / 2020$.

Nelms, S. E., E.M. Duncan, A.C. Broderick, T.S. Galloway, M.H. Godfrey, M. Hamann, P.K. Lindque and B.J. Godley. 2015. Plastic and marine turtles: a review and call for research. ICES J. Mar. Sci. , 73(2): 165-181.

Piedra-Castro, L. y V. Morales-Cerdas. 2015. Preferencias en la anidación de tortugas carey (Eretmochelys imbricata) y baulas (Dermochelys coriacea) en el Refugio Nacional de Vida Silvestre Gandoca Manzanillo, Limón, Costa Rica. Tecnol. Marcha, 28(3): 86-98.

Ramírez-Gallego, C. and K.G. Barrientos-Muñoz. 2020a. Illegal hawksbill trafficking: five years of records of the handicrafts and meat trades of the hawksbill turtle (Eretmochelys imbricata) in Cartagena de Indias, Colombia. J. Aquacult. Mar. Biol. , 9(3): 101-105. doi: 10.3389/fmars.2019.00817

Ramirez-Gallego, C. and K.G. Barrientos-Muñoz. 2021. Stopping illegal hawksbill trafficking in Cartagena de Indias, Colombia. Pp. 141-148. In: Sea turtle Research and Conservation: Lessons From Working In The Field. Editors, Nahill B. Elsevier Academic Press, USA. 224 p.Ramirez-Gallego, C. and K.G. Barrientos-Muñoz. 2020b. Sea Turtles at Serrana Island and Serranilla Island, Seaflower Biosphere Reserve, Colombian Caribbean. Front. Mar. Sci., 6: 817. doi: 10.3389/fmars.2019.00817

Reuter, A. and C. Allan. 2006. Tourists, t urtles and trinkets: a look at the trade in marine turtle products in the Dominican Republic and Colombia. Field Report, TRAFFIC, $12 \mathrm{p}$.

Rhodin, A. G., C.B. Stanford, P.P. Van Dijk, C. Eisemberg, L. Luiselli, R.A. Mittermeier, R. Hudson, B.D. Horne, E. Goode, G. Kuchling, A. Walde, E.H.W. Baard, K. H. Berry, A Bertolero, T.E.G. Blanck, R. Bour, K.A. Buhlmann, L.J. Cayot, S. Collett, A. Currylow, I. Das, T. Diagne, J.R. Ennen, G. Forero-Medina, M.G. Frankel, U. Fritz, G. García, J.W. Gibbons, P.M. Gibbons, S. Gong, J. Guntoro, M.D. Hofmeyr, J.B. Iverson, A.R. , Kiester, M. Lau, D.P. Lawson, J.E. Lovich, E.O., Moll, V.P. Páez, R. Palomo-Ramos, K. Platt, S.G. Platt, P.C.H. Pritchard, H.R. Quinn, S.C. Rahman, S. T. Randrianjafizanaka, J. Schaffer, W. Selman, H.B. Shaffer, D.S.K. Sharma, H. Shi, S. Singh, R. Spencer, K. Stannard, S. Sutcliffe, S. Thomson, and R. C. Vogt. 2018. Global conservation status of turtles and tortoises (order Testudines). Chel. Cons. Biol. , 17(2): 135-161. doi: 10.2744/CCB-1348.1 
Richardson, J.I., 1990. Estimation of S ea Turtle Abundance and Nesting Success on Mona Island, Puerto Rico. Final Rep. USFWS, Unit Coop. Agr. 1416- 009-1551, U.S. Fish and Wildlife Service. South Florida Ecosystem Office, Vero Beach, USA .

Rincón-Díaz, M.P. and C.J. Rodríguez-Zárate. 2004. Characterization of nesting beaches and feeding areas of marine turtles at the San Bernardo Archipelago, Colombian Caribbean. Bol. Invest. Mar. Cost. , 33(1): 137-158. doi: 10.25268/bimc.invemar.2004.33.0.252

Rincón, M.P., D. F. Rivera, C.J. Rodríguez y J.P. Tello. 2001. Establecimiento y caracterización estructural de puntos focales de anidación en el sector de Arrecifes, Parque Nacional Natural Tayrona, Caribe colombiano. Sem. I nvest., Univ. Bogotá Jorge Tadeo Lozano, Santa Marta. 67 p .

Rueda, J. V. 1987. Informe sobre la situación actual de las poblaciones de tortugas marinas en el Caribe Colombiano. Rep. Nal. Inderena II Simp. Tort. Mar. Atlántico Occidental STAO, Puerto Rico, 32 p.

SIRAP Caribe e Invemar . 2013. Articulación del Subsistema de Áreas Marinas Protegidas (SAMP) al plan de acción del SIRAP Caribe. Ser. Publ. Gen. Invemar, $.38 \mathrm{p}$.

Suganuma, H., 2005. Leatherback turtle management of feral pig predation in Indonesia: 37-38. In: Kinan, I. (Ed.), Proc. ${ }^{\text {nd }}$ West. Pac. Sea Turtle Coop. Res. Manag. Workshop Vol. 1 . West. Pac. Reg. Fish. Manag. Counc., Honolulu.

Ulloa, J.C., Quiroga, D.A. y T.R. Ruiz. 2016. Caracterización de los p rincipales humedales costeros en la jurisdicción de Carsucre. Inf. Téc. , Carsucre. Sincelejo. $79 \mathrm{p}$.

Whytlaw, P.A., W. Edwards and B.C. Congdon. 2013. Marine turtle nest depredation by feral pigs (Sus scrofa) on the w estern Cape York Peninsula, Australia: implications for management. Wildl. Res., 40: 377-384.

RECIBIDO/RECEIVED: 26/07/2020

ACEPTADO/ACCEPTED: 25/05/2021 